\title{
Genetic analysis of atherosclerosis identifies a major susceptibility locus in the major histocompatibility complex of mice
}

\author{
Andrew T. Grainger ${ }^{\# a}$, Michael B. Jones ${ }^{\# b}$, Jing Li $^{\mathrm{b}}$, Mei-Hua Chen $^{\mathrm{b}}$, Ani Manichaikul ${ }^{\mathrm{c}}$, and \\ Weibin Shia,b, ${ }^{*}$ \\ aDepartment of Biochemistry \& Molecular Genetics, University of Virginia, Charlottesville, VA, \\ USA \\ bDepartment of Radiology and Medical Imaging, University of Virginia, Charlottesville, VA, USA \\ 'Department of Public Health Sciences, University of Virginia, Charlottesville, VA, USA \\ \# These authors contributed equally to this work.
}

\begin{abstract}
Background and aims: Recent genome-wide association studies (GWAS) have identified over 50 significant loci containing common variants associated with coronary artery disease. However, these variants explain only $26 \%$ of the genetic heritability of the disease, suggesting that many more variants remain to be discovered. Here, we examined the genetic basis underlying the marked difference between SM/J-Apoe $e^{-/-}$and BALB/cJ-Apoe ${ }^{-/}$mice in atherosclerotic lesion formation.
\end{abstract}

Methods: 206 female $\mathrm{F}_{2}$ mice generated from an intercross between the two $\mathrm{Apoe}^{-/-}$strains were fed 12 weeks of western diet. Atherosclerotic lesion sizes in the aortic root were measured and 149 genetic markers genotyped across the entire genome.

Results: A significant locus, named Ath49(LOD score: 4.18), for atherosclerosis was mapped to the $\mathrm{H} 2$ complex [mouse major histocompatibility complex (MHC)] on chromosome 17. Bioinformatic analysis identified 12 probable candidate genes, including Tnfrsf 21, Adgrf1, Adgrf5, Mep1a, and Pla2g7. Corresponding human genomic regions of Ath49 showed significant association with coronary heart disease. Five suggestive loci on chromosomes 1, 4, 5, and 8 for atherosclerosis were also identified. Atherosclerotic lesion sizes were significantly correlated with HDL but not with non-HDL cholesterol, triglyceride or glucose levels in the $\mathrm{F}_{2}$ cohort.

Conclusions: We have identified the MHC as a major genetic determinant of atherosclerosis, highlighting the importance of inflammation in atherogenesis.

*Corresponding author. University of Virginia, Snyder Bldg Rm 266, 480 Ray C. Hunt Dr, P.O. Box 801339, Fontaine Research Park, Charlottesville, VA, 22908, USA. ws4v@ virginia.edu (W. Shi).

Conflict of interest

Authors declared no conflict of interest, including financial, personal or other relationships with other people or organizations, within the past three years that could have inappropriately influenced the work reported here. 


\section{Keywords}

Atherosclerosis; Major histocompatibility complex; Quantitative trait locus; Mice

\section{Introduction}

Atherosclerosis, the primary cause of heart attack, ischemic stroke and peripheral arterial disease, is a complex disease resulting from interactions between environmental and genetic factors [1]. The important role of genetic factors in atherosclerosis has been clearly demonstrated in numerous studies, including prospective studies of twins, families and cohorts [2-4], and genome-wide association studies (GWAS) [5-7]. Apart from rare cases of Mendelian inheritance that are caused by missense or nonsense mutations with large effect, the vast majority of coronary heart disease is complex, probably involving many genes of small effect [8]. Recent meta-analysis of GWAS data have identified $>50$ loci harboring common variants significantly associated with coronary heart disease [5-7]. Together they only explain $26 \%$ of the genetic heritability of coronary heart disease [5], suggesting that many more loci have not been discovered. Furthermore, most of the loci identified have small effect sizes with odds ratios $(\mathrm{OR})<1.25$ [5]; thus it's extremely challenging to establish causality between a genetic variant and disease in humans.

A complementary approach to finding genes and pathways involved in atherosclerosis is to study animal models. This allows for strict control over environmental influence and accurate phenotypic characterization of atherosclerotic lesions. Apolipo-protein E-null $\left(\right.$ Apoe $\left.{ }^{--}\right)$and LDL receptor-null ( $\mathrm{Lldl}^{-r^{-}}$) mouse models reproduce all phases of atherosclerotic lesions seen in humans [9,10]. Over a dozen intercrosses or backcrosses have been generated from atherosclerosis-susceptible and -resistant inbred strains with either Apoe ${ }^{-/-}$or $\mathrm{LIdlI}^{-1-}$ background, leading to identification of 50 unique atherosclerosis susceptibility loci (http://www.informatics.jax.org/allele). Unfortunately, nearly all the crosses generated have chosen C57BL/6 (B6) mice as an atherosclerosis-susceptible strain; thus limiting their mapping power and coverage of allelic diversity. Creation of new genetic crosses using a different susceptible strain may discover new loci and also empower bioinformatics analysis for finding causative genes. When a QTL for the same trait has been mapped to the same chromosomal location with multiple crosses derived from different inbred strains, whole-genome sequences and SNP databases available for them can be utilized to prioritize candidate genes. We recently have shown that SM/J (SM) and SWR/J Apoe ${ }^{-/}$mice are susceptible to atherosclerosis compared to BALB/cJ (BALB) or C3H/HeJ Apoe ${ }^{-/}$mice [11]. In this study, we performed QTL analysis using a female $\mathrm{F}_{2}$ cohort derived from an intercross between BALB/cJ (BALB)- $A p o e^{-/-}$and SM- $A p o e^{-/-}$mice to understand the genetic control of atherosclerosis susceptibility.

\section{Materials and methods}

\subsection{Mice}

BALB-Apoe ${ }^{--}$and SM- $A$ poe $^{-/-}$mice were made in our laboratory using the congenic breeding method as previously reported [11]. The creation of a female $\mathrm{F}_{2}$ cohort from the 
two Apoe ${ }^{-/}$strains was recently described [12]. The animals were weaned at 3 weeks of age, and at 6 weeks of age switched onto a Western diet. After 12 weeks of Western diet, mice were euthanized for assessment of atherosclerotic lesion formation in the aorta.

\subsection{Quantitation of aortic atherosclerosis}

Atherosclerotic lesion areas in the aortic root of mice were measured as previously reported [13]. Lesion areas were measured on oil red $\mathrm{O}$ stained sections using Zeiss AxioVision 4.8 software. The eight largest lesion areas were added up for each mouse and this sum was used for statistical analysis.

\subsection{Measurements of plasma glucose and lipid levels}

Plasma total cholesterol, HDL cholesterol, triglyceride and glucose levels were measured using commercial kits as reported [14]. Non-HDL cholesterol was calculated as the difference between total and HDL cholesterol.

\subsection{Genotyping}

$F_{2}$ mice were genotyped using the Illumina LD linkage panel, as reported [12].

Microsatellite markers were typed by PCR for regions of chromosome 8 that were not covered by informative SNP markers. A total of 149 markers were included in QTL analysis.

\subsection{Statistical analysis}

QTL analysis was performed using J/qtl and MapManager QTX software, as reported [12]. LOD threshold values were determined from 1000 permutations of the observed data, which are provided in Data-in-Brief.

\subsection{Human genetic association analysis}

Human homologous genomic regions corresponding to mouse QTL intervals were examined for associations with coronary heart disease using the dataset from the

CARDIoGRAMplusC4D Consortium that included 60,801 coronary artery disease cases and 123,504 controls [5]. Web-based software LocusZoom was used to determine associations of SNPs in regions of interest with coronary artery disease.

\subsection{Prioritization of candidate genes}

Bioinformatic tools were used to prioritize candidate genes for significant atherosclerosis QTL that was mapped in two or more crosses derived from different parental strains. Likely candidate genes were defined as those containing a non-synonymous SNP in a coding region or a SNP in upstream regulatory region, and this SNP was shared by the parental strains carrying the high allele but different from the one shared by the parental strains carrying the low allele of a QTL, as reported [15]. Analysis was performed using a combination of SNP sources, including the Sanger Mouse Genomes Project, Mouse Phenome Database, and Ensembl. Web-based software RaptorX was used to predict the potential impact of an amino acid substitution on the 3D structure of protein product (http://raptorx.uchicago.edu/ Structure_Prediction/predict). 


\subsection{Gene expression analysis}

mRNA expression levels of potential candidate genes in the thymus of BALB mice, which carry the $\mathrm{H} 2^{\mathrm{d}}$ haplotype, and $\mathrm{C} 3 \mathrm{H} / \mathrm{HeJ}$ mice, which have the $\mathrm{H} 2^{\mathrm{k}}$ haplotype, were determined by real-time quantitative PCR. RNA extraction, cDNA preparation and PCR amplification were performed as we previously reported [16]. The following forward and reverse primers were used for Tnfrsf 21 : (5'- TTGAAGCTTGTAGCAGCCCA-3') 5'TATCCATTGGAGAAGGCCGC-3'; Tdrd6: 5'-CATCGAAAACCCTGGCTCCT-3' 5'-GAGATGGCTCGCTGCTTTTG-3'; Mep1a: 5'-TCTGGGCACGCCTTTTTCTA-3'\% 5'-GAAGATCAAGCCAGCGATGC-3'; Pla2g7: 5'-CAGCTTGGAGCTGTCAGGAG-3'/ 5'-TGAGCATACAGCCTCCTCGT-3'; Adgrf5: 5'-GCAGCACTTACACGCTCAAG-3' 5'-CTCTGGCTCCATAGGCACTG-3'; Adgrf1: 5'-ACAGCCTCCAGGGTGACTAGA-3'/5'-GTGAAGAGAGGGACGAGCCA-3'; Gapdh: 5'-GAGGCCGGTGCTGAGTATGT-3'/5'-AAGGGTGGAGCCAAAAGGGTCATC-3'). PCR reactions were run in triplicate for each sample. The transcript level of each gene was analyzed using BioRad CFX Manager 3.1 software and expressed as a comparative cycle threshold $(\Delta \Delta \mathrm{Ct})$ value relative to the level of Gapdh. PCR products amplified with fewer than 30 cycles were electrophoresed on agarose gel to evaluate PCR efficiency.

\section{Results}

\subsection{QTL analysis of atherosclerotic lesions}

Values of atherosclerotic lesion areas in 206 female $\mathrm{F}_{2}$ mice were calculated by summing up the top eight sections for each mouse. These values display a normal distribution (Fig. 1). Genome-wide QTL analysis of these data revealed one significant QTL on chromosome 17 and five suggestive QTLs on chromosomes 1, 4, 5, and 8 for atherosclerosis (Fig. 2). Details of the QTLs detected, including locus name, LOD score, peak location, 95\% confidence interval (CI), genome-wide $\mathrm{P}$ value, high allele, and mode of inheritance, are shown in Table 1. The QTL on chromosome 17 had a significant LOD score of 4.18 and peaked at 26.08 cM. It exerted effect in a dominant mode of inheritance with the BALB allele conferring susceptibility and the SM allele conferring resistance to atherosclerosis (Fig. 3A, Table 2). This QTL was overlapping in the confidence interval with Ath26, mapped in an AKRApoe $^{--} \times$DBA-Apoe ${ }^{-/-}$intercross [17]. Because this QTL was mapped in an intercross derived from distinct parental strains, it was named Ath49 in accordance to the guideline provided by the International Committee on Standardized Genetic Nomenclature for Mice (http://www.informatics.jax.org/mgihome/ nomen/gene.shtml). The QTL on chromosome 1 had a suggestive LOD score of 2.28 and peaked at $97.02 \mathrm{cM}$ (Fig. 3B). This QTL replicated Ath1, mapped in several crosses [18-21]. The QTL on chromosome 4 peaked at $71.37 \mathrm{cM}$ and had a LOD score of 2.52. It replicated Athsq1 mapped in a (MOLF/Ei $\times$ B6- $\left.L d l r^{-/-}\right) \times$ B6- $L d l r^{-1-}$ backcross [22]. Two suggestive QTLs were detected on chromosome 5, peaking at 34.19 and $69.4 \mathrm{cM}$, respectively (Figs. 2 and 3C). The distal QTL replicated Ath42, mapped in a B6-Apoe ${ }^{-/-} \times$BALB-Apoe $e^{-/-}$intercross [19]. The proximal QTL replicated a suggestive one mapped in a B6-Apoe $e^{-/} \times \mathrm{C} 3 \mathrm{H}-$ Apoe $^{-/-}$intercross [20] and was named Ath50. The QTL on chromosome 8 peaked at $46.14 \mathrm{cM}$ and had a suggestive LOD score of 2.18. It was partially overlapping with $A t h 40$ mapped in a B $6 \times \mathrm{BALB} / \mathrm{cByJ} L D L R^{-1-}$ intercross [23]. SM alleles were responsible for increased lesion sizes for the chromo-some 
1, 5 and 8 QTLs but decreased lesion sizes for Ath49. The chromosome 4 QTL affected lesion formation in a heterotic manner as $\mathrm{F}_{2}$ mice with heterozygous alleles exhibited increased lesion size over those with homozygous alleles.

\subsection{Candidate genes for Ath 49}

The QTL for atherosclerosis on chromosome 17 has also been mapped in an AKR-Apoe ${ }^{-/} \times$ DBA/2-Apoe $e^{-1-}$ intercross with the DBA/2 allele conferring susceptibility and the AKR allele conferring resistance to atherosclerosis [17]. The high allele strains BALB and DBA/2 share the same $\mathrm{H} 2{ }^{\mathrm{d}}$ haplotype, which falls within the $A t h 49$ interval, whereas the low allele strains $\mathrm{SM}$ and AKR have either $\mathrm{H} 2^{\mathrm{v}}$ or $\mathrm{H} 2^{\mathrm{k}}$ haplotype. To find candidate genes for the QTL, we searched the Sanger SNP database for genes containing nonsynonymous SNP(s) or SNP(s) in upstream regulatory regions that are shared by the high allele strains (BALB, $\mathrm{DBA} / 2$ ) but are different from ones carried by the low allele strain (AKR) in the confidence interval. SM strain was not included because its genomic sequences for the region were incomplete. 12 candidate genes were identified. For these genes, we further searched other publicly available databases, including the Mouse Phenome Database (http:// phenome.jax.org/db/q?rtnsnp/home) and Ensembl (http://www.ensembl.org/index.html), and compiled the results in Table 3. Of them, Tnfrsf21, Adgrf1, Adgrf5, Mep1a, and Tdrd6 are located precisely underneath the linkage peak and contain one or more nonsynonymous SNPs leading to amino acid substitutions. SIFT (Sorting Intolerant From Tolerant) score, a parameter for estimating the impact of an amino acid substitution on protein function, suggest that the function of protein products encoded by Adgrf1, Adgrf5, and Mep1a is highly likely different between the high and low allele strains (Table 3). Mepla contains over a dozen non-synonymous SNPs between the high and low allele strains. Three of them had extremely low SIFT scores, including substitutions at amino acid residues $247 \mathrm{~F} / \mathrm{Y}$ (score: 0.01), $232 \mathrm{~N} / \mathrm{S}$ (score: 0.02) and $201 \mathrm{H} / \mathrm{Y}$ (score: 0.06). We further used online software RaptorX to predict the impact of multiple amino substitutions on the 3D structure of Mep1a protein product (http://raptorx.uchicago.edu/StructurePrediction/). The predicted 3D structure of Mepla protein was noticeably different between the high allele strain BALB and the low allele strain AKR (Fig. 4).

The expression of Tnfrsf21, Adgrf1, Adgrf5, Mep1a, Pla2g7, and Tdrd6 in the thymus of BALB and $\mathrm{C} 3 \mathrm{H} / \mathrm{HeJ}$ mice were determined by real-time PCR. $\mathrm{C} 3 \mathrm{H} / \mathrm{HeJ}$ mice have the same $\mathrm{H} 2{ }^{\mathrm{k}}$ haplotype as AKR mice. The transcript level of each gene was normalized to the level of reference gene Gapdh. Adgrfl and Mepla expression levels were significantly lower in BALB than in $\mathrm{C} 3 \mathrm{H} / \mathrm{HeJ}$ mice $(p<0.05$ ) (Fig. 5A). Tdrd6 expression level was also lower in BALB mice although the difference was not significant $(p=0.059)$. Noticeable differences for these three genes were observed between the two strains on agarose gel electrophoreses of PCR products amplified with fewer than 30 cycles (Fig. 5B). No significant differences in expression levels were found for Tnfrsf21, Adgrf5, and Pla2g7.

\subsection{Association with coronary artery disease in humans}

The confidence interval of Ath 49 extended from 43 to $63 \mathrm{Mb}$, as denoted by the histogram of interval mapping graph (Fig. 3A). The corresponding human syntenic region spans approximately from 30 to $55 \mathrm{Mb}$ on chromosome 6 . We searched the human GWAS meta- 
analysis dataset for variants in this region that were associated with coronary artery disease. Multiple SNPs, particularly those in the 39-40 Mb region, showed significant associations with the disease $\left(p<3.3 \times 10^{-4}\right)$ (Fig. 6). The lead SNP rs56336142 had a highly significant $p$ value of $1.85 \times 10^{-8}$. Genes adjacent to this SNP include KCNK5, KCNK16, KCNK17, and SAYSD1. The mouse counterparts of these genes are located on mouse chromosome 14 and thus they are the causal genes of Ath49.

\subsection{Correlations of atherosclerotic lesion sizes with plasma lipid and glucose levels}

The associations of atherosclerotic lesion sizes with fasting plasma lipid and glucose levels were evaluated using the $F_{2}$ population (Fig. 7). A significantly inverse correlation with HDL cholesterol levels was observed $(\mathrm{r}=-0.188 ; p=0.0068)$. Those $\mathrm{F} 2$ mice with higher HDL cholesterol levels tended to develop smaller atherosclerotic lesions than those with lower levels. Nevertheless, HDL only accounted for $3.5 \%$ of the variance in atherosclerotic lesion sizes of the $\mathrm{F}_{2}$ population, as denoted by the $r^{2}$ value, a measure of the correlation between the two variables. No significant correlations were found with plasma levels of nonHDL cholesterol $(r=0.022 ; p=0.757)$, triglyceride $(R=-0.093 ; p=0.184)$, or glucose $(r=$ $0.00021 ; p=0.998)$.

\section{Discussion}

In this study, we performed QTL analysis using a female cohort derived from an intercross between BALB and SM Apoe ${ }^{-1-}$ mouse strains and identified one significant QTL (Ath49) on chromosome 17 and six suggestive QTLs on chromosomes 1, 4, 5, and 8 for atherosclerotic lesion sizes in the aorta. We then used bioinformatic tools to reduce the number of candidate genes for Ath49 down to a dozen. The corresponding human homologous region of Ath49 showed significant associations with coronary artery disease. Moreover, plasma HDL cholesterol was found to explain 3.5\% of the variance in lesion sizes of the $\mathrm{F}_{2}$ population.

Ath26 is an atherosclerosis susceptibility QTL on chromosome 17, initially mapped in an intercross between AKR-Apoe ${ }^{-/-}$and DBA/2-Apoe ${ }^{-/-}$mice [17]. In that cross, the QTL peaked at 36 or $47 \mathrm{Mb}$, depending on whether or not the lesion data were log-transferred. The confidence interval of Ath 26 overlaps with the $\mathrm{H} 2$ complex (mouse major histocompatibility complex). The present study also mapped an atherosclerosis susceptibility QTL to the same region, but because it was mapped with a cross derived from distinct parental strains, the QTL was given a new designation, Ath49, according to the guideline established by the International Committee on Standardized Genetic Nomenclature for Mice (http://www.informatics.jax.org/mgihome/nomen/gene.shtml). Direct evidence supporting an importance role for the $\mathrm{H} 2$ complex in regulation of atherosclerosis susceptibility comes from our study of C3.SW- $H 2^{b} A p o e^{-1-}$ mice [24]. C3H/HeJ mice are extremely resistant to atherosclerosis even on the Apoe null background. However, after their $H 2^{k}$ allele was replaced with $H 2^{b}$ allele and bone marrow was reconstituted with syngeneic or allogenic marrow, these mice exhibited a 21-fold increase in atherosclerotic lesion size [24].

By examining genes containing SNPs that were shared among the high allele strains (BALB, $\mathrm{DBA} / 2$ ) but different from those carried by the low allele strain (AKR), we were able to 
reduce the number of candidates down to 12 genes in the Ath49 interval. This analysis was based on the observation that genetic variations between mouse strains are primarily inherited from their progenitorsM. m. musculus and M. m. domesticus [25]. Mouse strains carrying an allele that results in a high value for a given trait often share the same ancestral allele harboring the underlying causal gene, while strains carrying an allele that produces a low value for the trait share a different ancestral allele [26]. A limitation of this analysis was that the causal gene would be missed if the QTL arose from a more recent mutation. Because QTL arises from changes in the function or the quantity of a gene product, we focused on genes that carry a nonsynonymous coding SNP or a SNP in the upstream regulatory region segregating between the high allele and low allele strains of the QTL crosses. Tnfrsf21, Adgrf1, Adgrf5, Mep1a, Pla2g7 and Tdrd6 are located underneath the linkage peak of Ath49, and contains one or more nonsynonymous SNPs or a SNP in the upstream regulatory region segregating between the high allele and low allele parental strains.

Genes in the $\mathrm{H} 2$ complex encode proteins that are directly involved in or indirectly associated with antigen presentation through interactions with $\mathrm{T}$ lymphocytes. The thymus is the primary lymphoid organ of the immune system where T lymphocytes mature; thus we compared the expression of above prioritized candidate genes in this organ of BALB and $\mathrm{C} 3 \mathrm{H} / \mathrm{HeJ}$ mice. $\mathrm{C} 3 \mathrm{H} / \mathrm{HeJ}$ mice carry the same $\mathrm{H} 2{ }^{\mathrm{k}}$ haplotype as AKR mice, and BALB mice share the same $\mathrm{H} 2{ }^{\mathrm{d}}$ haplotype with $\mathrm{DBA} / 2$ mice. The differential expression in an important immune issue between the high and low allele strains further support the probability for Adgrf1 and Mep1a to be the underlying QTL genes. Moreover, both genes contain one or more nonsynonymous SNPs with deleterious SIFT scores that almost certainly affect protein function. Mepla encodes the a subunit of meprin metalloproteinase, and the absence of this gene aggravates chronic inflammation in the colons of mice [27]. Mepla probably also exerts an anti-inflammatory role in the arterial wall; thus its low expression or protein malfunction is expected to accelerate inflammatory responses and atherosclerosis in the high allele mouse strains.

Association of the MHC complex with coronary artery disease has been observed in recent human GWAS studies [5,27]. The present analysis of human GWAS meta-analysis dataset also revealed multiple variants in the $\mathrm{MHC}$ region associated with coronary artery disease. KCNK5 has been suggested to be an underlying causal gene contributing to coronary artery disease [5]. However, as KCNK5 is located on mouse chromosome 14 but not 17, obviously it is not the causal genes of Ath49. Furthermore, the chromosomal region harboring KCNK5 has only shown a suggestive linkage with atherosclerotic lesions [28].

In this study, we identified multiple suggestive QTLs for atherosclerotic lesion sizes, including the ones on chromosome 5. The LOD score plots for chromosome 5 revealed two distinct peaks, suggesting the existence of two QTLs for atherosclerosis. The distal QTL was overlapping with Ath42 mapped in a B6 $\times$ BALB Apoe ${ }^{-/-}$intercross [19]. The proximal QTL replicated a suggestive QTL mapped in a $\mathrm{B} 6 \times \mathrm{C} 3 \mathrm{H}$ Apoe $^{-/-}$intercross [20] and was named Ath50. Naming a suggestive locus is considered appropriate if it is repeatedly observed [29]. Promising candidate genes for this QTL include Lrrc66, Pdgfra, Epha5, Cenpc1, Uba6, and Tmprss11g. These genes are located underneath the linkage peak, and 
contains one or more SNPs segregating between the high allele and low allele parental strains of the QTL crosses (data not shown). Moreover, the human syntenic region harboring these genes has been shown to be associated with coronary heart disease [5].

A slight but statistically significant correlation was observed between HDL cholesterol levels and aortic atherosclerotic lesion sizes in the $\mathrm{F}_{2}$ cross. Similar findings have been observed with other mouse crosses $[15,23,30]$. These findings are consistent with the observation made from Mendelian randomization studies of HDL for role in human coronary heart disease [31,32]. Although Mendelian randomization studies of blood lipids have suggested a role for triglyceride and LDL cholesterol in human coronary heart disease, no significant correlations with atherosclerotic lesions were observed in the present cross or previous crosses $[13,33]$.

In summary, we have identified a major QTL for atherosclerosis in the mouse MHC region using a segregating $\mathrm{F}_{2}$ population, and further applied bioinformatic tools to define underlying candidate genes. As the MHC region harbors genes that play an important role in both innate and adaptive immunity, our findings highlight the significance of inflammation in atherogenesis and its potential for developing anti-atherosclerotic therapy.

\section{Acknowledgments}

Financial support

This work was supported by NIH grants DK097120 and HL112281.

\section{References}

[1]. Mozaffarian D, Benjamin EJ, Go AS, Arnett DK, Blaha MJ, Cushman M, et al., Heart disease and stroke statisticse2015 update: a report from the American Heart Association, Circulation 131 (2015) e29-322. [PubMed: 25520374]

[2]. Marenberg ME, Risch N, Berkman LF, Floderus B, de Faire U, Genetic susceptibility to death from coronary heart disease in a study of twins, N. Engl. J. Med 330 (1994) 1041-1046. [PubMed: 8127331]

[3]. Lloyd-Jones DM, Nam BH, D’Agostino S, Levy D, Murabito JM, Wang TJ, et al., Parental cardiovascular disease as a risk factor for cardiovascular disease in middle-aged adults: a prospective study of parents and offspring, JAMA 291 (2004) 2204-2211. [PubMed: 15138242]

[4]. Bachmann JM, Willis BL, Ayers CR, Khera A, Berry JD, Association between family history and coronary heart disease death across long-term follow-up in men: the Cooper Center Longitudinal Study, Circulation 125 (2012) 3092-3098. [PubMed: 22623718]

[5]. Nikpay M, Goel A, Won HH, Hall LM, Willenborg C, Kanoni S, et al., A comprehensive 1,000 Genomes-based genome-wide association meta-analysis of coronary artery disease, Nat. Genet 47 (2015) 1121-1130. [PubMed: 26343387]

[6]. CARDIoGRAMplusC4D Consortium, Deloukas P, Kanoni S, Willenborg C, Farrall M, Assimes TL, et al., Large-scale association analysis identifies new risk loci for coronary artery disease, Nat. Genet 45 (2013) 25-33. [PubMed: 23202125]

[7]. Coronary Artery Disease (C4D), Genetics Consortium, A genome-wide association study in Europeans and South Asians identifies five new loci for coronary artery disease, Nat. Genet 43 (2011) 339-344. [PubMed: 21378988]

[8]. Welch C, Lusis AJ, Genetics of common forms of heart disease: a long and winding road, Circ. Res 113 (2013) 1035-1036. [PubMed: 24115066] 
[9]. Nakashima Y, Plump AS, Raines EW, Breslow JL, Ross R, ApoE-deficient mice develop lesions of all phases of atherosclerosis throughout the arterial tree, Arterioscler. Thromb 14 (1994) 133140. [PubMed: 8274468]

[10]. Ishibashi S, Goldstein JL, Brown MS, Herz J, Burns DK, Massive xanthomatosis and atherosclerosis in cholesterol-fed low density lipoprotein receptor-negative mice, J. Clin. Investig 93 (1994) 1885-1893. [PubMed: 8182121]

[11]. Liu S, Li J, Chen MH, Liu Z, Shi W, Variation in type 2 diabetes-related phenotypes among apolipoprotein E-deficient mouse strains, PLoS One 10 (2015) e0120935. [PubMed: 25946019]

[12]. Wang Q, Grainger AT, Manichaikul A, Farber E, Onengut-Gumuscu S,Shi W, Genetic linkage of hyperglycemia and dyslipidemia in an intercross between BALB/cJ and SM/J Apoe-deficient mouse strains, BMC Genet 16 (2015) 133. [PubMed: 26555648]

[13]. Su Z, Li Y, James JC, McDuffie M, Matsumoto AH, Helm GA, et al., Quantitative trait locus analysis of atherosclerosis in an intercross between $\mathrm{C} 57 \mathrm{BL} / 6$ and $\mathrm{C} 3 \mathrm{H}$ mice carrying the mutant apolipoprotein E gene, Genetics 172 (2006) 1799-1807. [PubMed: 16387874]

[14]. Shi W, Wang Q, Choi W, Li J, Mapping and congenic dissection of Genetic Loci contributing to hyperglycemia and dyslipidemia in mice, PLoS One 11 (2016) e0148462. [PubMed: 26859786]

[15]. Rowlan JS, Li Q, Manichaikul A, Wang Q, Matsumoto AH, Shi W, Atherosclerosis susceptibility Loci identified in an extremely atherosclerosis-resistant mouse strain, J. Am. Heart Assoc 2 (2013) e000260. [PubMed: 23938286]

[16]. Manichaikul A, Wang Q, Shi YL, Zhang Z, Leitinger N, Shi W, Characterization of Ath29, a major mouse atherosclerosis susceptibility locus, and identification of Rcn2 as a novel regulator of cytokine expression, Am. J. Physiol. Heart Circ. Physiol 301 (2011) H1056-H1061. [PubMed: 21666121]

[17]. Smith JD, Bhasin JM, Baglione J, Settle M, Xu Y, Barnard J, Atherosclerosis susceptibility loci identified from a strain intercross of apolipoprotein E-deficient mice via a high-density genome scan, Arterioscler. Thromb. Vasc. Biol 26 (2006) 597-603. [PubMed: 16373612]

[18]. Wang X, Ria M, Kelmenson PM, Eriksson P, Higgins DC, Samnegard A, et al., Positional identification of TNFSF4, encoding OX40 ligand, as a gene that influences atherosclerosis susceptibility, Nat. Genet 37 (2005) 365-372. [PubMed: 15750594]

[19]. Zhang Z, Rowlan JS, Wang Q, Shi W, Genetic analysis of atherosclerosis and glucose homeostasis in an intercross between C57BL/6 and BALB/cJ apolipo-protein E-deficient mice, Circ. Cardiovasc Genet 5 (2012) 190-201. [PubMed: 22294616]

[20]. Wang SS, Schadt EE, Wang H, Wang X, Ingram-Drake L, Shi W, et al., Identification of pathways for atherosclerosis in mice: integration of quantitative trait locus analysis and global gene expression data, Circ. Res 101 (2007) e11-30. [PubMed: 17641228]

[21]. Tomita H, Zhilicheva S, Kim S, Maeda N, Aortic arch curvature and atherosclerosis have overlapping quantitative trait loci in a cross between 129S6/SvEvTac and C57BL/6J apolipoprotein E-null mice, Circ. Res 106 (2010) 1052-1060. [PubMed: 20133902]

[22]. Welch CL, Bretschger S, Latib N, Bezouevski M, Guo Y, Pleskac N, et al., Localization of atherosclerosis susceptibility loci to chromosomes 4 and 6 using the Ldlr knockout mouse model, Proc. Natl. Acad. Sci. U. S. A 98 (2001) 7946-7951. [PubMed: 11438740]

[23]. Burkhardt R, Sundermann S, Ludwig D, Ceglarek U, Holdt LM, Thiery J, et al., Cosegregation of aortic root atherosclerosis and intermediate lipid phenotypes on chromosomes 2 and 8 in an intercross of C57BL/6 and BALBc/ByJ low-density lipoprotein receptor-/- mice, Arterioscler. Thromb. Vasc. Biol 31 (2011) 775-784. [PubMed: 21252064]

[24]. Shi W, Zhang Z, Chen MH, Angle JF, Matsumoto AH, Genes within the MHC region have a dramatic influence on radiation-enhanced atherosclerosis in mice, Circ. Cardiovasc Genet 3 (2010) 409-413. [PubMed: 20729504]

[25]. Wade CM, Kulbokas EJ, 3rd, Kirby AW, Zody MC, Mullikin JC, Lander ES, et al., The mosaic structure of variation in the laboratory mouse genome, Nature 420 (2002) 574-578. [PubMed: 12466852]

[26]. Peters LL, Robledo RF, Bult CJ, Churchill GA, Paigen BJ, Svenson KL, The mouse as a model for human biology: a resource guide for complex trait analysis, Nat. Rev. Genet 8 (2007) 58-69. [PubMed: 17173058] 
[27]. Banerjee S, Jin G, Bradley SG, Matters GL, Gailey RD, Crisman JM, et al., Balance of meprin A and $\mathrm{B}$ in mice affects the progression of experimental inflammatory bowel disease, Am. J. Physiol. Gastrointest. Liver Physiol 300 (2011) G273-G282. [PubMed: 21071511]

[28]. Dansky HM, Shu P, Donavan M, Montagno J, Nagle DL, Smutko JS, et al., A phenotypesensitizing Apoe-deficient genetic background reveals novel atherosclerosis predisposition loci in the mouse, Genetics 160 (2002) 1599-1608. [PubMed: 11973313]

[29]. Abiola O, Angel JM, Avner P, Bachmanov AA, Belknap JK, Bennett B, et al., The nature and identification of quantitative trait loci: a community's view, Nat. Rev. Genet 4 (2003) 911-916. [PubMed: 14634638]

[30]. Rowlan JS, Zhang Z, Wang Q, Fang Y, Shi W, New quantitative trait loci for carotid atherosclerosis identified in an intercross derived from apolipoprotein E-deficient mouse strains, Physiol. Genomics 45 (2013) 332-342. [PubMed: 23463770]

[31]. Holmes MV, Asselbergs FW, Palmer TM, Drenos F, Lanktree MB, Nelson CP, et al., Mendelian randomization of blood lipids for coronary heart disease, Eur. Heart J 36 (2015) 539-550. [PubMed: 24474739]

[32]. White J, Sofat R, Hemani G, Shah T, Engmann J, Dale C, et al., Plasma urate concentration and risk of coronary heart disease: a Mendelian randomisation analysis, Lancet Diabetes Endocrinol 4 (2016) 327-336. [PubMed: 26781229]

[33]. Li Q, Li Y, Zhang Z, Gilbert TR, Matsumoto AH, Dobrin SE, et al., Quantitative trait locus analysis of carotid atherosclerosis in an intercross between C57BL/6 and $\mathrm{C} 3 \mathrm{H}$ apolipoprotein Edeficient mice, Stroke 39 (2008) 166-173. [PubMed: 18048852] 


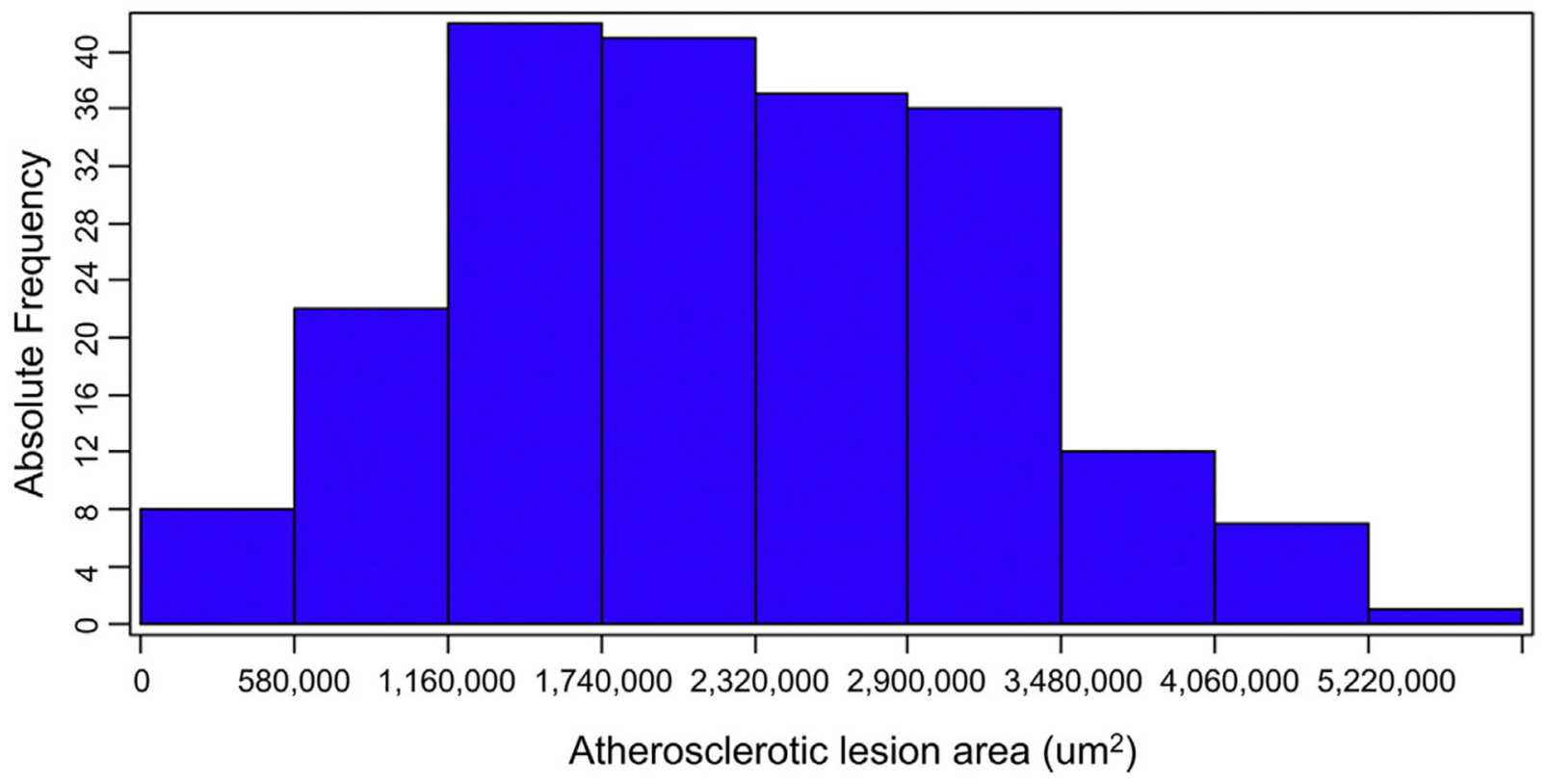

Fig. 1. Frequency distributions of atherosclerotic lesion sizes in 206 female $F_{2}$ mice derived from BALB-Apoe ${ }^{-/-}$and SM-Apoe ${ }^{-/-}$mice.

The lesion size of each mouse was the sum of lesion areas on 8 cross-sections with the largest readings. The graph was created using a plotting function of J/qtl software. 


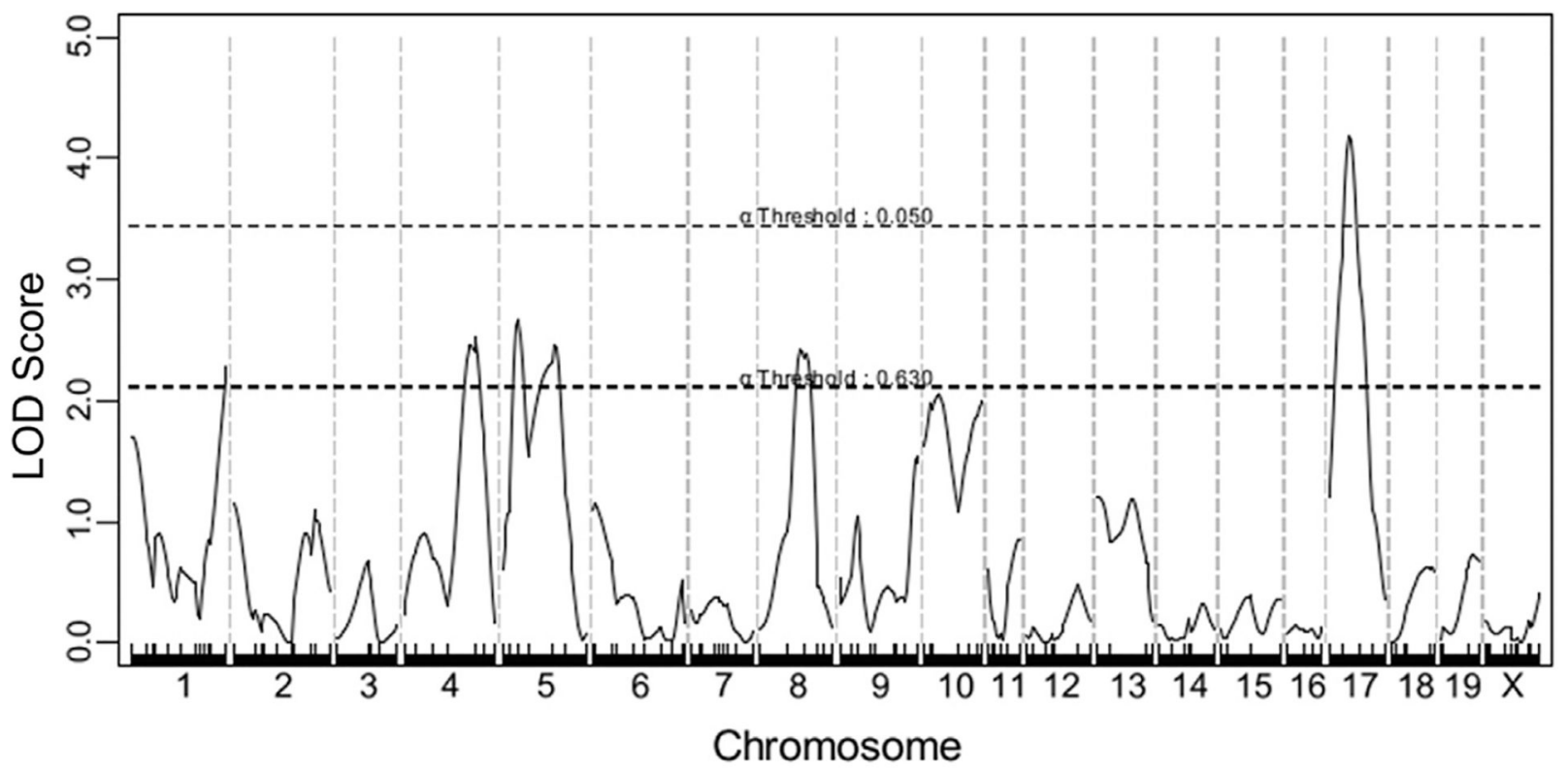

Fig. 2. A genome-wide scan to search for main effect QTLs influencing atherosclerotic lesion sizes in the $\mathbf{F}_{2}$ cohort.

Chromosomes 1 through $\mathrm{X}$ are represented numerically on the $\mathrm{X}$-axis. Each minor tick on the $\mathrm{X}$ axis represents one informative genetic marker. The Y-axis represents the LOD score. Two horizontal dashed lines denote genome-wide thresholds for suggestive $(P=0.63)$ and significant $(P=0.05)$ linkage. 


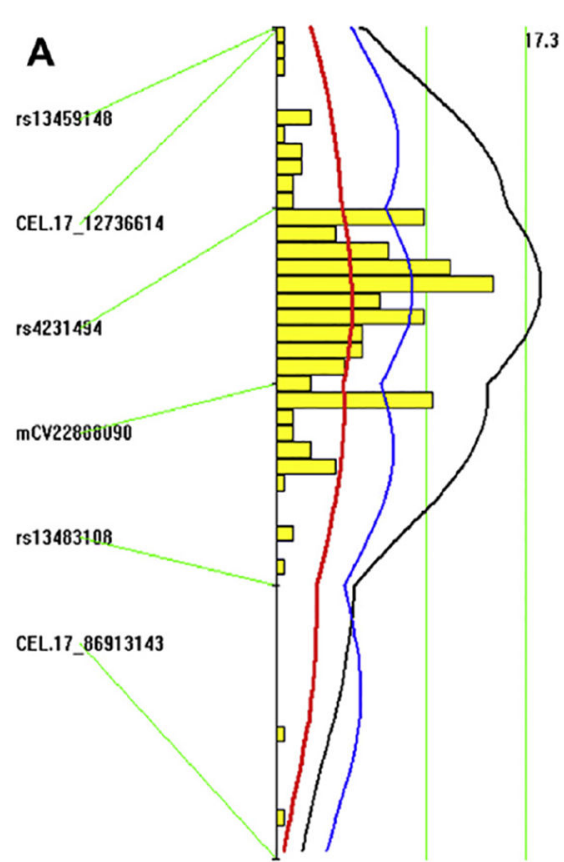

Chr 17

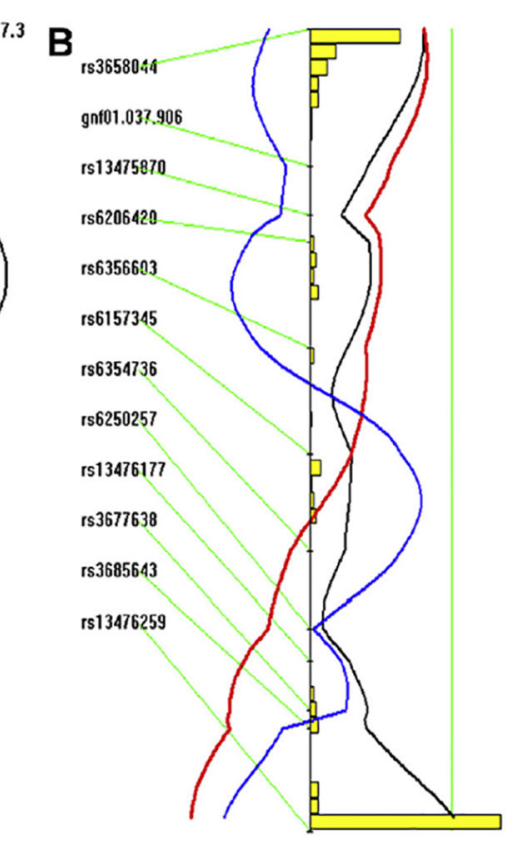

Chr 1

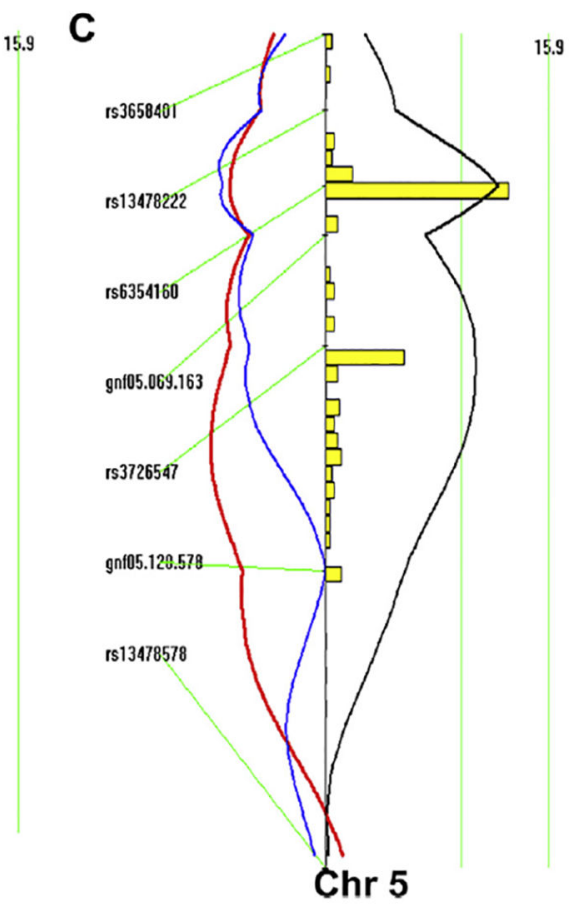

Fig. 3. Interval mapping graphs for atherosclerotic lesion sizes plotted by MapManager QTX. $\mathrm{A}$, chromosomes 17; $\mathrm{B}$, chromosome 1 ; and $\mathrm{C}$, chromosome 5 . The black line represents LOD scores calculated at a 1-cM interval, the blue plot represents the effect of BALB allele, and the red plot represents the effect of SM allele. The histogram estimates the confidence interval of a QTL. Two green vertical lines represent genome-wide LOD thresholds for suggestive (left line) and significant linkage (right line). (For interpretation of the references to colour in this figure legend, the reader is referred to the web version of this article.) 

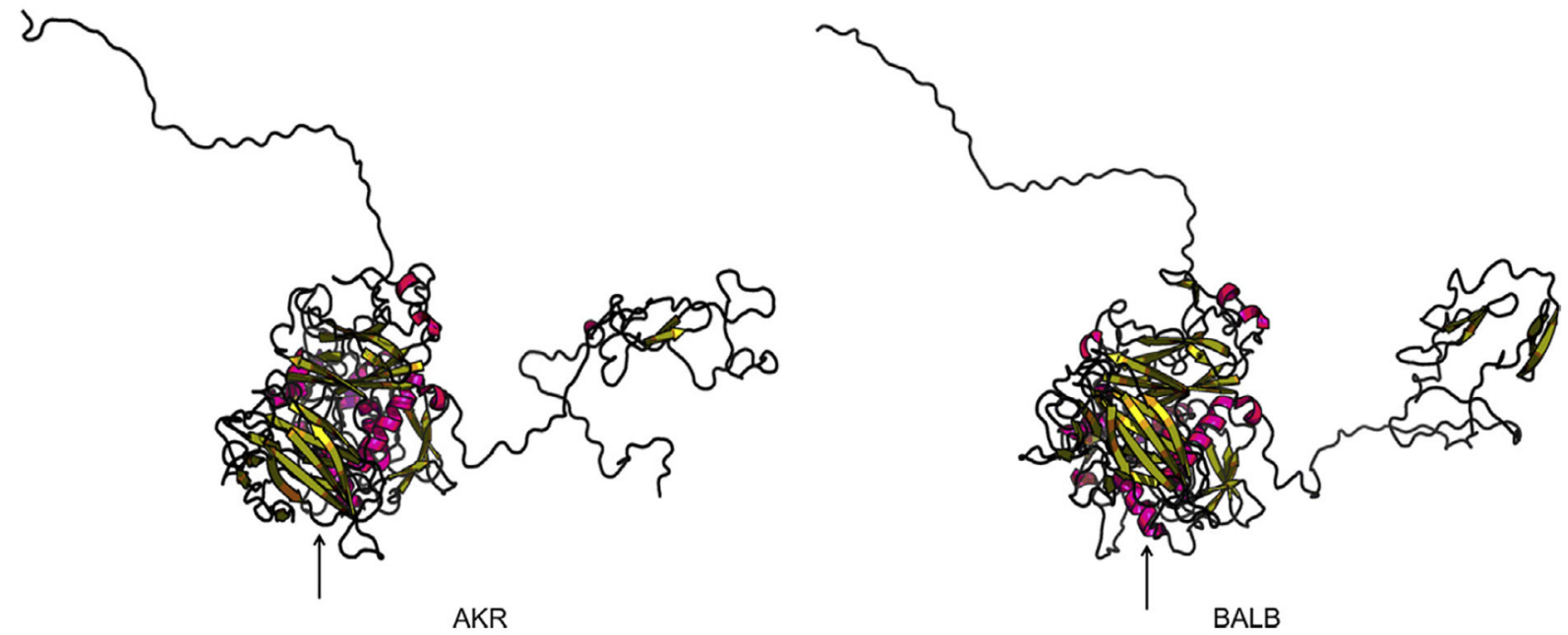

Fig. 4. Predicted 3D structure of Mep1a protein plotted by RaptorX software.

Left panel: AKR mouse, and right panel: BALB mouse. The predicted 3D structure is noticeably different between the two mouse strains, specifically for the region pointed by the arrow. 
A

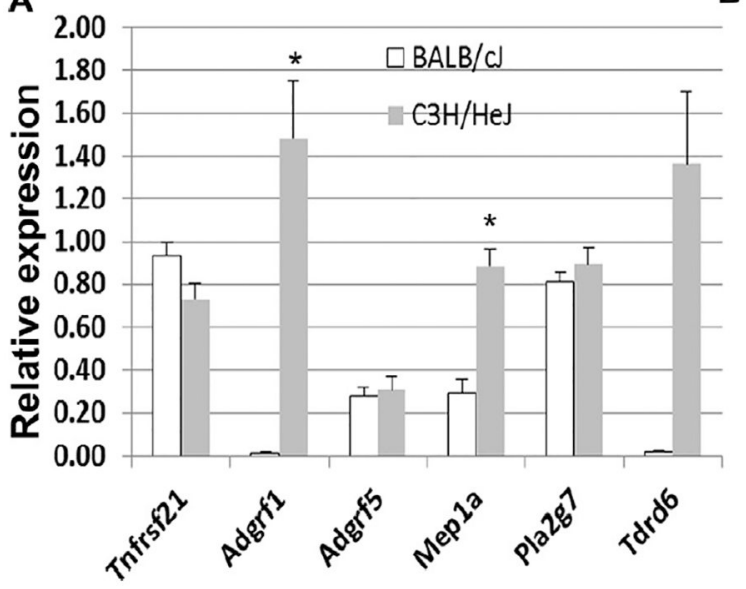

B

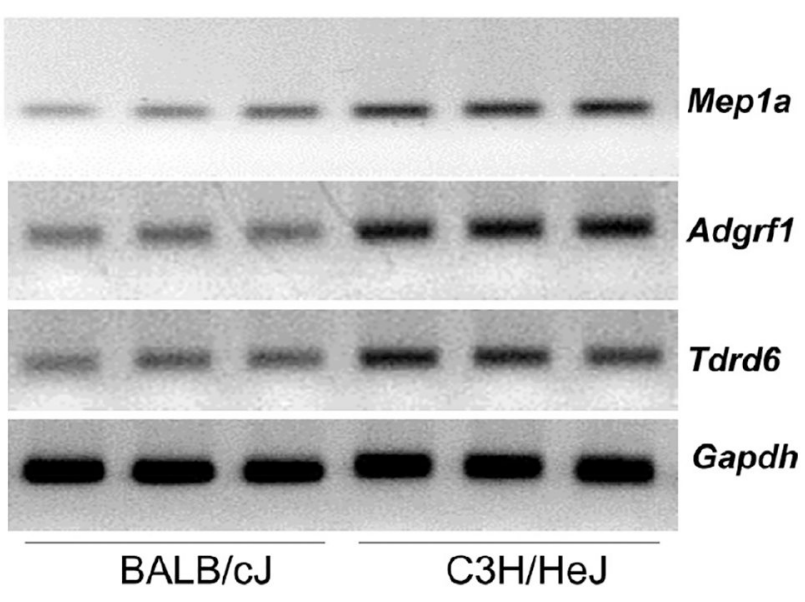

Fig. 5. Expression of candidate genes in the thymus of BALB and $\mathrm{C} 3 \mathrm{H} / \mathrm{HeJ}$ mice fed a chow diet. A, real-time PCR analysis of candidate gene expression. Results are expressed as comparative cycle threshold $(\Delta \Delta \mathrm{Ct})$ values normalized by the reference gene Gapdh. Values are means $\pm \mathrm{SE}$ of $3 \mathrm{e} 4$ biological replicates of each strain. $* P<0.05$. B, semi-quantitative PCR analyses of gene expression in the thymus of BALB and C3H/HeJ mice. Each lane represents an individual mouse. 


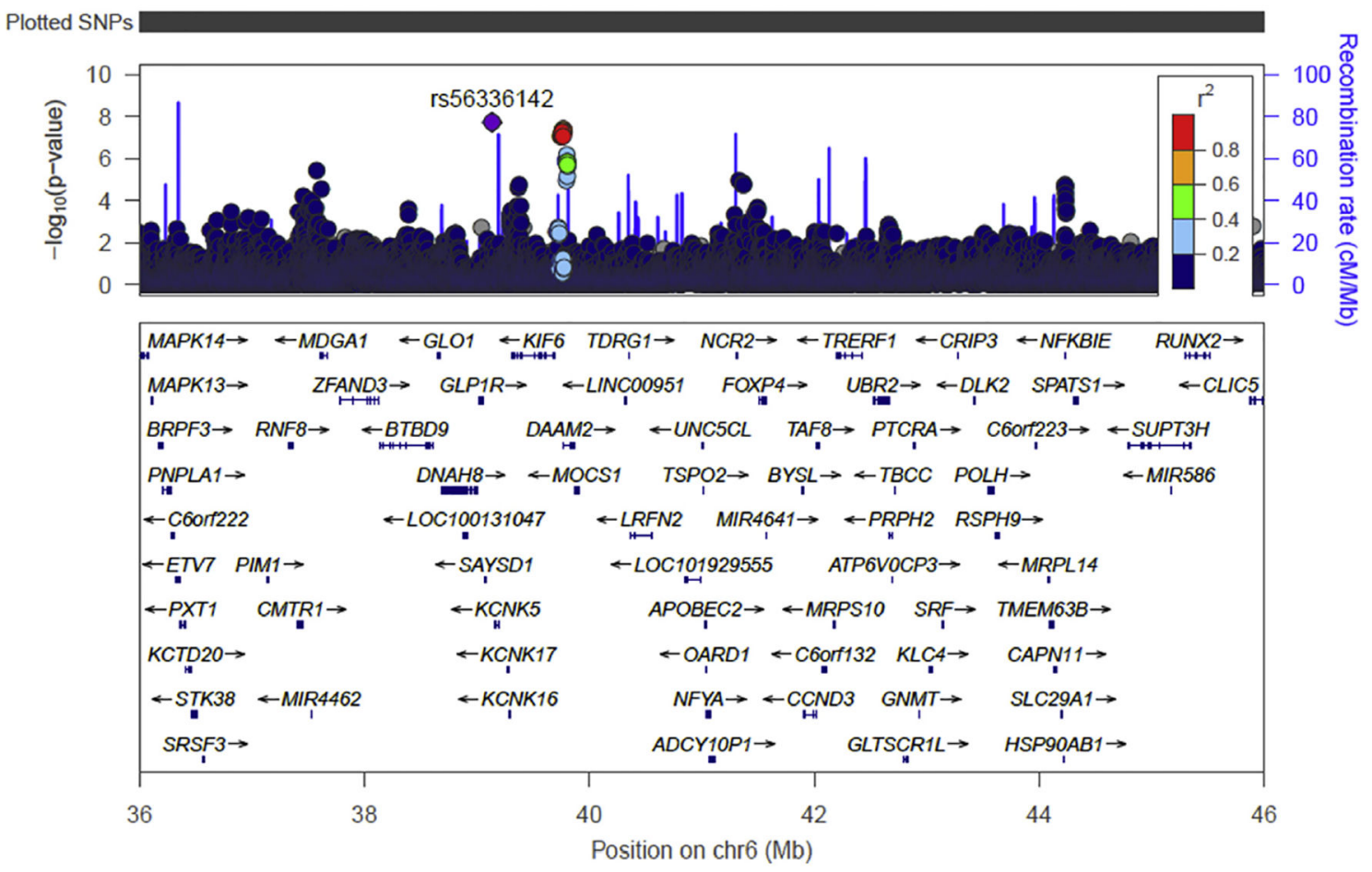

Fig. 6. Regional association plot for coronary heart disease using the dataset from the CARDIoGRAMplusC4D Consortium.

Linkage disequilibrium (LD) was inferred based on the 1000 Genomes Phase 3 EUR reference panel. 

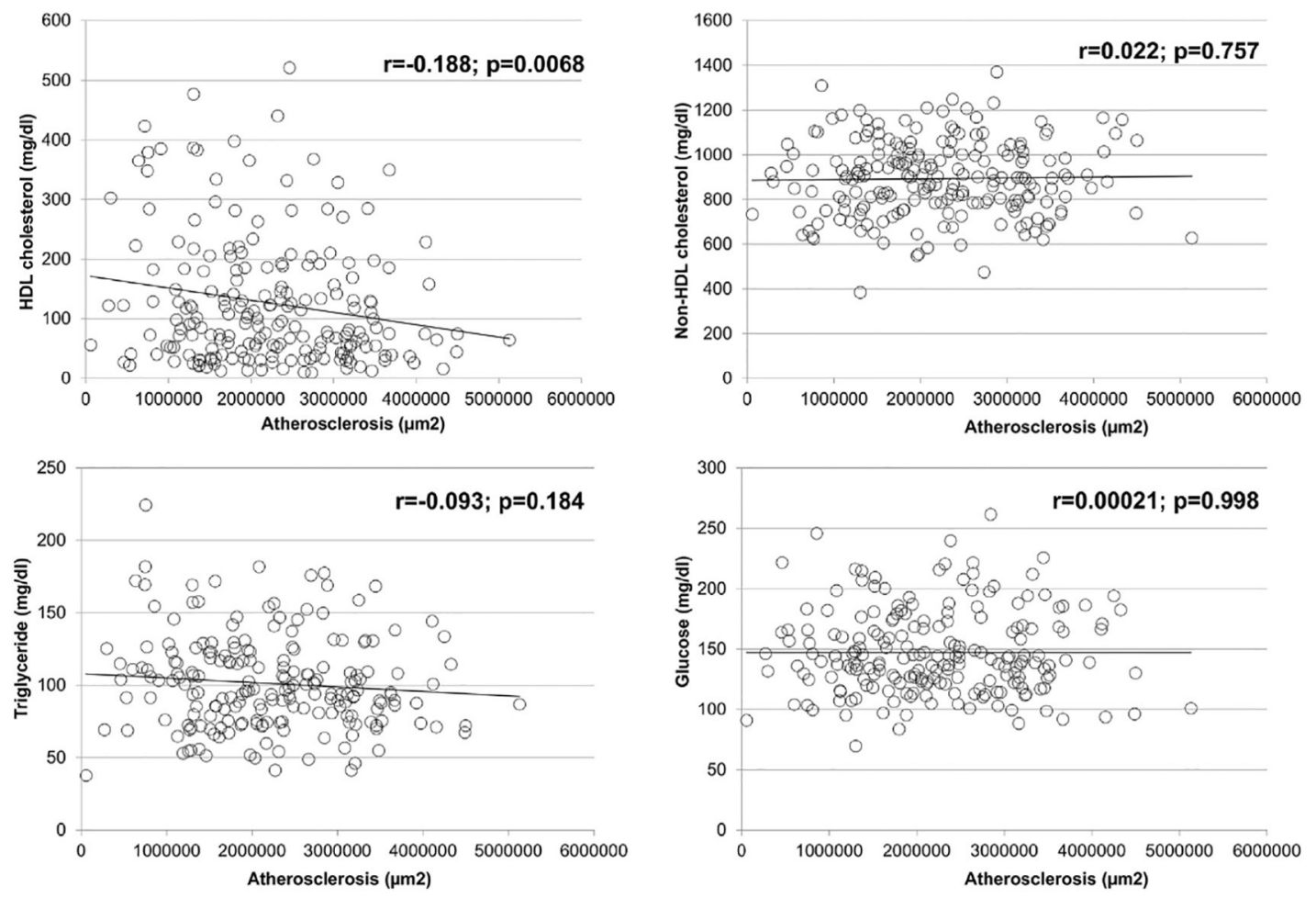

Fig. 7.

Correlations of atherosclerotic lesion sizes with plasma levels of HDL, non-HDL cholesterol, triglyceride and glucose in the $\mathrm{F}_{2}$ population. Each point represents values of an individual $\mathrm{F}_{2}$ mouse. The correlation coefficient $(r)$ and significance $(p)$ are shown. 


\section{롤 \\ 길}

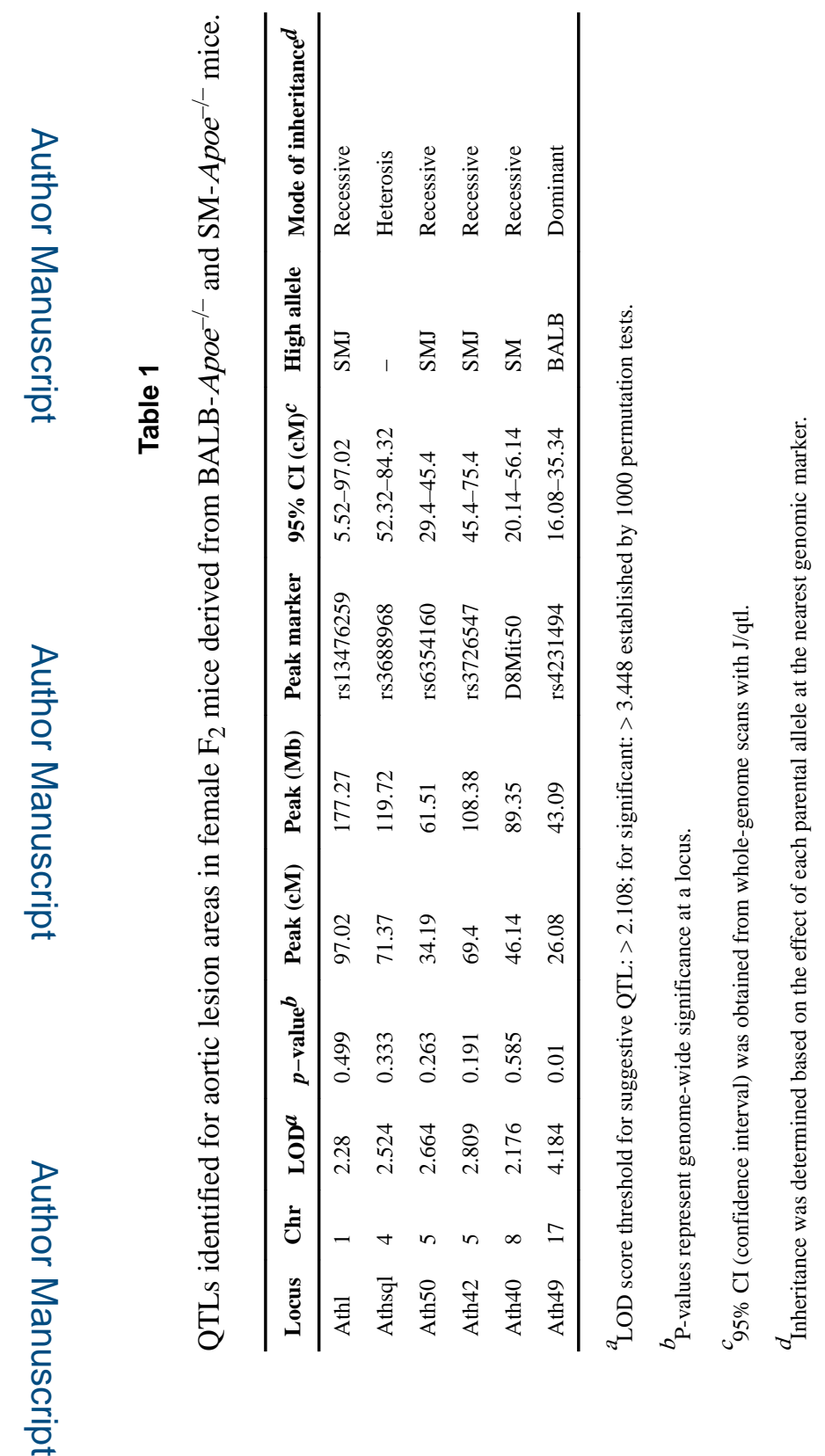

Atherosclerosis. Author manuscript; available in PMC 2018 October 10. 


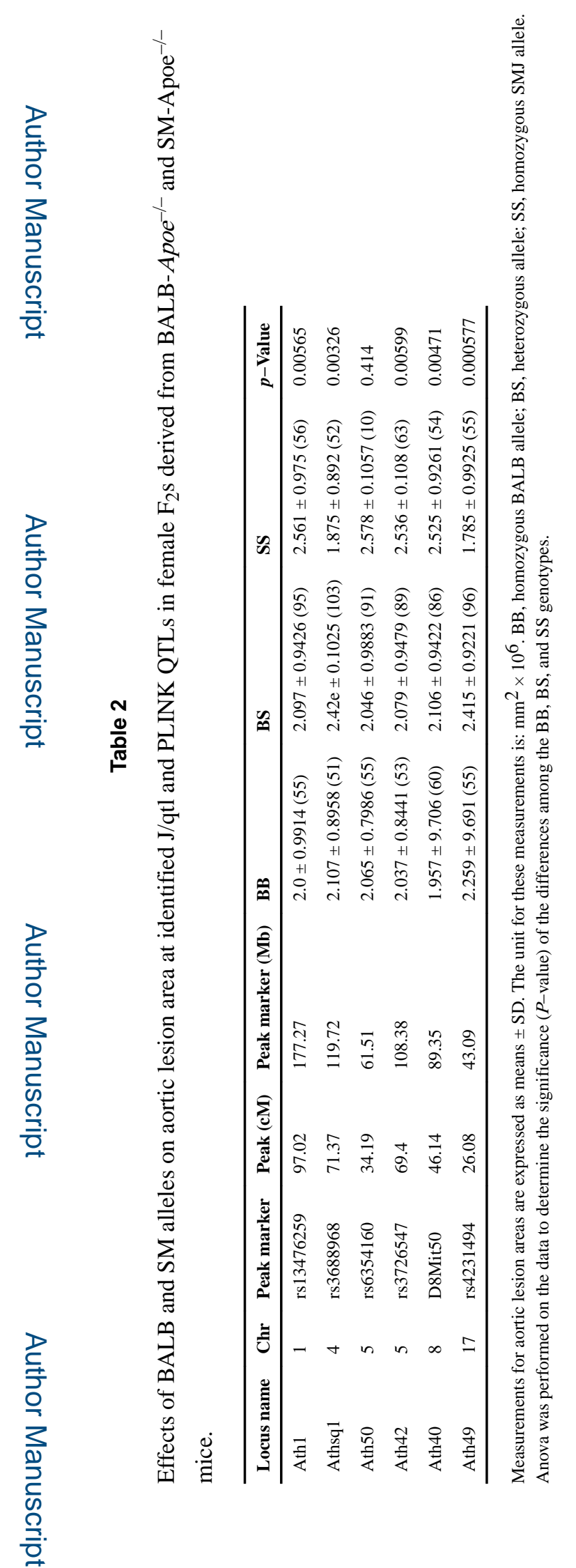

Atherosclerosis. Author manuscript; available in PMC 2018 October 10. 


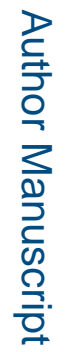

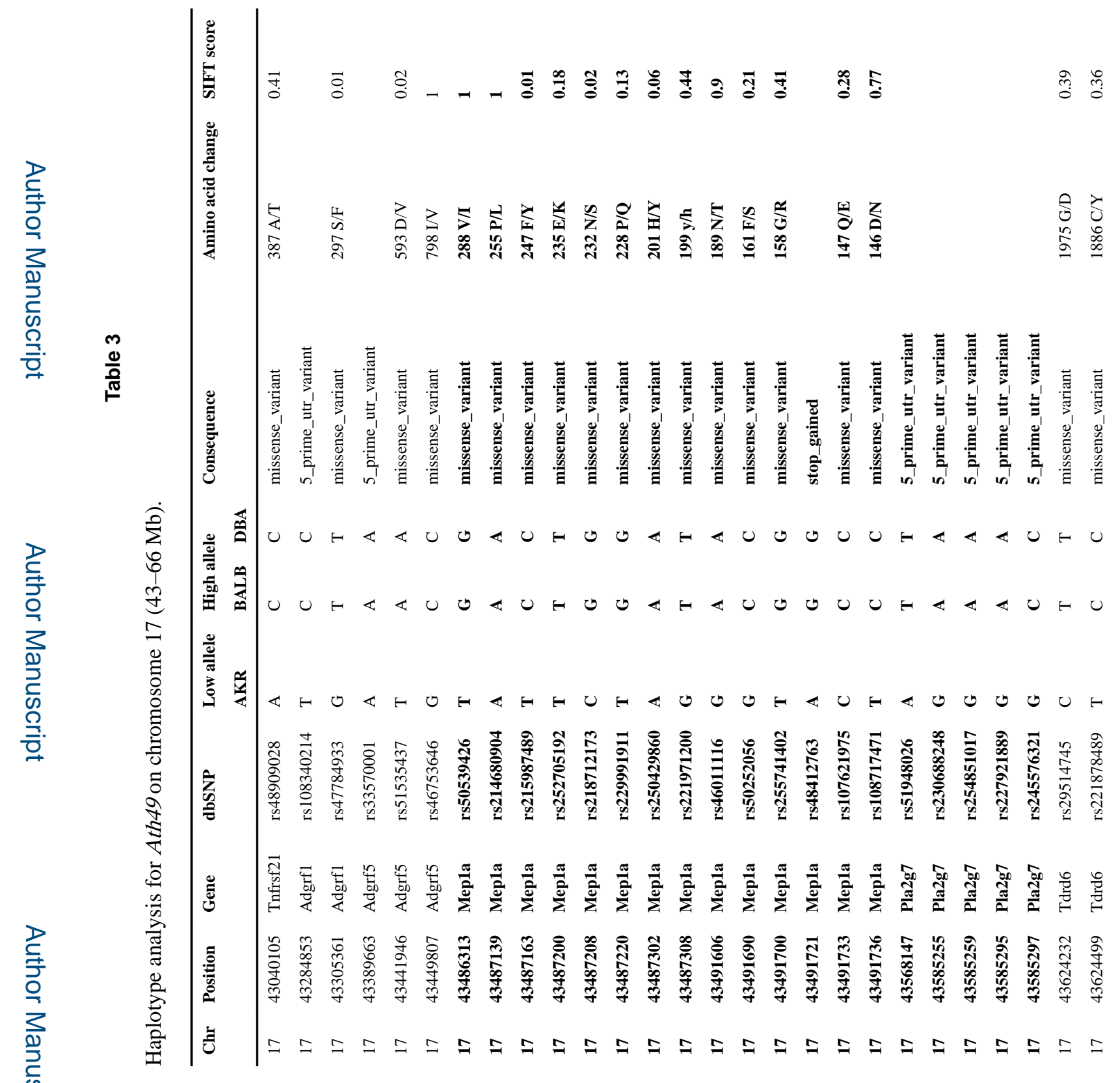

Atherosclerosis. Author manuscript; available in PMC 2018 October 10. 


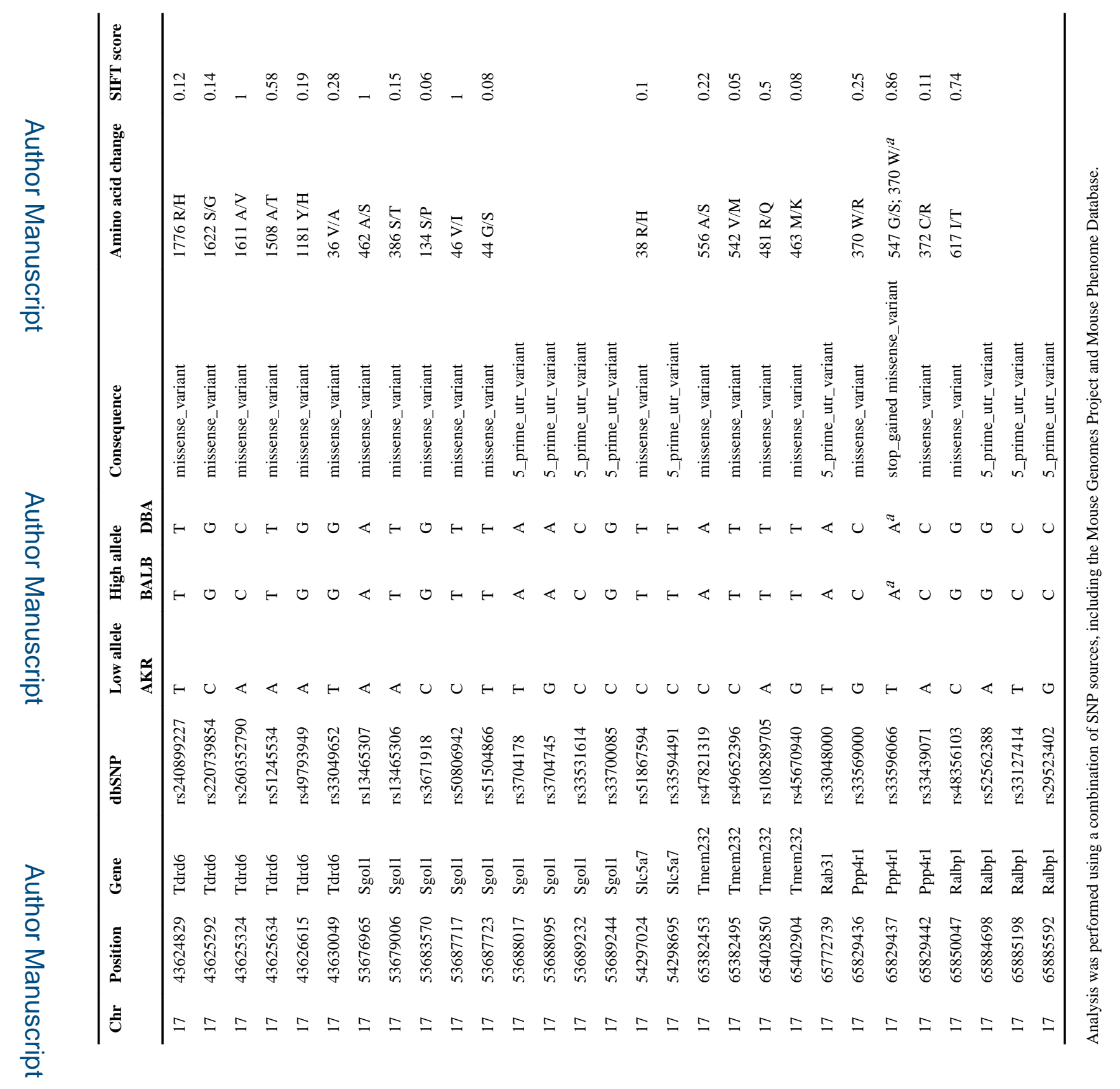

로을

Atherosclerosis. Author manuscript; available in PMC 2018 October 10. 


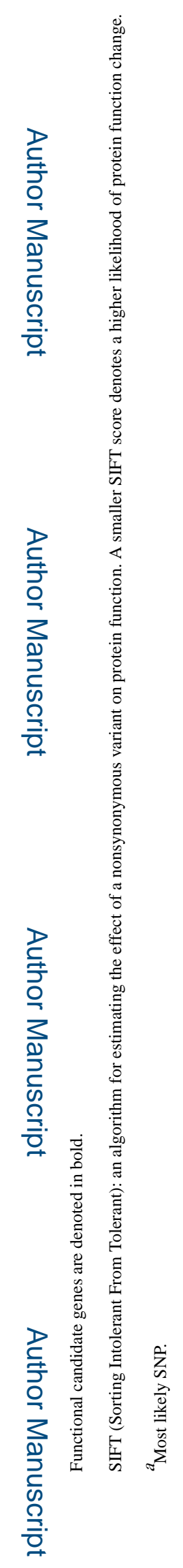

Atherosclerosis. Author manuscript; available in PMC 2018 October 10. 